\title{
THIẾP LẬP BẢNG ĐIỀU KHIỂN KẾT HợP CỦA Bộ CHỈ SỐ CHẤT LƯợNG CỐT LÕI MỖI TUẦN BẰNG NGÔN NGỮ R
}

\author{
Đỗ Văn Niệm, Lê Thị Châu, Lê Thị Thu Thúy \\ Bệnh viện Nhi Đồng 1
}

\begin{abstract}
TÓM TẮT
Giới thiệu: Chỉ số là phương pháp đo lường chất lượng cơ bản và hình thức báo cáo phù hợp sẽ cung cấp thêm thông tin đểnhà quản trị ra quyết định. Phương pháp: Chuẩn hóa dữ liệu giám sát quá trình và đo lường kết quả hiện có thành dữ liệu mảng. Sử dụng kết quả này để phát triển bảng điêu khiển kết hợp bằng ngôn ngữ R (gói phân tích: flexdashboard, knitr, tidyverse, readxl, reshape2, lubridate, qicharts2, qcc, ggQC, gridExtra, plotly, DT), với định dạng .html nhằm dễ phổ biến. Người dùng được hỏi ý kiến để hoàn thiện thiết kếnhằm đảm bảo sự tiện lợi, đủ thông tin cân thiết, khả năng truy vết nguồn gốc vấn đề và dễ tìm kiếm thông tin. Kết quảa: Bảng điêu khiển chỉ số gồm: tóm tắt chỉ số tuần và trung bình năm hiện hành, biểu đồ kiểm soát từng nhóm chỉ số theo tuần so sánh với năm liền kề trước, biểu đồ Pareto chỉ rõ nội dung ít tuân thủ hoạc nhóm sự kiện bất lợi chiếm đa số, phân tích chỉ số theo khoa và quy trình bằng biểu đồ lưới để đối sánh. Bảng dữ liệu tổng hợp về tỷ lệ tuân thủ theo khoa và quy trình mỗi tuân, kết hợp chức năng tìm kiếm giúp người dùng truy cập nhanh thông tin. Khảo sát ý kiến người dùng 2 lần trong tiến trình phát triển có kết quả đánh giá phù hợp và dễ hiểu trên $75 \%$. Kết luận: Bảng điều khiển chỉ số không những giúp nhà quản trị bệnh viện dễ thấy bức tranh toàn cảnh về chất lượng, mà còn cung cấp đủ thông tin chi tiết để mỗi khoa, phòng thực hiện cải tiến.
\end{abstract}

Từ khóa: Bảng điều khiển kết hợp, chỉ số chất lượng, ngôn ngữ $R$.

\section{ABSTRACT \\ ESTABLISHING A WEEKLY COMPACT DASHBOARD OF KEY QUALITY INDICATORS BASED ON R LANGUAGE}

Do Van Niem, Le Thi Chau, Le Thi Thu Thuy

Introduction: Quality indicator is an essential methodology for quality measurements and its suitable presentation format supplies hospital managers more information for making decisions. Method: Existing data on process supervision and outcome measurements were standardized and transformed to panel format which were then utilizied to develop a compact dashboard based on $\mathrm{R}$ language (packages were used: flexdashboard, knitr, tidyverse, readxl, reshape2, lubridate, qicharts2, qcc, ggQC, gridExtra, plotly, DT) and .html layout for releasing easily. Users were asked for their needs and comments to revise the design, and guarantee that the dashboard was user-friendly, supplied enough necessary information, allowed for tracing the origin of problems and searching information easily. Results: Contents of the dashboard included a summary of weekly and current year's indicators, weekly indicator control charts by groups accompanied with the last year's one.

Nhận bài: 20-1-2021; Chấp nhận: 15-4-2021

Người chịu trách nhiệm chính: Đỗ Văn Niệm

Địa chỉ: ĐT: 0909997987; Email: niemdv@nhidong.org.vn 
Pareto diagram indicated most non-compliance steps and negative feedback events, gridplotting analysis by departments and procedures for benchmarking. A weekly summary data table of compliance proportion by departments and procedures with searching option helped users accessing information rapidly. Two surveys were deployed during designing and developing dashboard period that had the result of more than $75 \%$ user-accepted.

Conclusion: The compact dashboard not only helps hospital managers easily capture the overview of service qualities but also supply enough information for every department to make decisions for quality improvement.

Keywords: Compact dashboard, quality indicators, R language.

\section{1. ĐặT VẤN ĐỀ}

Chỉ số là phương pháp đo lường chất lượng cơ bản và hình thức báo cáo phù hợp sẽ cung cấp thêm thông tin để nhà quản trị ra quyết định. Khi số lượng chỉ số còn ít, việc báo cáo và đọc kết quả khá đơn giản, dưới hình thức bản in hoặc tệp báo cáo định dạng văn bản có chứa bảng số liệu và biểu đồ. Nếu số lượng chỉ số đủ lớn (10 hoặc nhiều hơn), nhất là cần phân tầng khi phân tích thì phương pháp truyền thống có nhiều bất lợi, làm mất thời gian của người báo cáo và cũng khó khăn cho người sử dụng trong quá trình tổng hợp thông tin đầu vào để ra quyết định. Bệnh viện Nhi Đồng 1 đã triển khai 42 chỉ số, có 6 chỉ số trực tiếp trên hệ thống thông tin bệnh viện (HIS: Hospital Information System), chỉ số tuân thủ quy trình kỹ thuật được phân nhóm theo người thực hiện và kỹ thuật cụ thể. Phân tầng phân tích dữ liệu theo khoa, quy trình kỹ thuật và bước ít được tuân thủ là cần thiết nhằm giúp nhà quản lý nhanh chóng xác định các ưu tiên theo dõi và can thiệp tiếp theo.

Theo quy định pháp lý và xu hướng quản lý chất lượng hiện nay, [1,2,3] việc thiết lập bảng điều khiển tích hợp nhiều chỉ số là rất cần thiết nhằm hỗ trợ kịp thời cho các nhà quản trị trong quá trình truy vết các vấn đề chất lượng và ra quyết định.

Trong lĩnh vực khoa học về dữ liệu, có 2 ngôn ngữ lập trình phổ biến và phát triển mạnh hiện nay là $R$ và Python. Nếu Python có ưu thế khi phân tích dữ liệu lớn, thì thế mạnh của $R$ là phân tích biểu đồ và xử lý dữ liệu thống kê. $R$ là ngôn ngữ sử dụng phổ biến trong nghiên cứu khoa học, vì vậy việc tiếp cận nó ở các bệnh viện sẽ đơn giản và dễ dàng hơn so với Python, vì người dùng không phải học thêm một ngôn ngữ khác. R là ngôn ngữ mã nguồn mở, miễn phí, hiện tại đã có nhiều gói phân tích chuyên dùng, có thể thực hiện hầu hết phân tích thống kê, biểu đồ cơ bản và chuyên sâu trong quản lý chất lượng. Dù $\mathrm{R}$ là ngôn ngữ mã nguồn mở và miễn phí, cộng đồng $R$ (gồm các chuyên gia hàng đầu về thống kê) thường xuyên phát triển, bình duyệt các gói phân tích và cảnh báo những lỗi tiềm ẩn có thể sai lệch, nhờ đó có thể đảm bảo chất lượng kết quả phân tích.[4]

Bảng điều khiển chỉ số có thể thiết kế qua 2 hướng chính: một là trình bày trên một website gồm một trang chủ với các trang liên kết cho các chỉ số thành phần, hai là bảng điều khiển kết hợp (compact) gồm trang tổng hợp với các thẻ (tab) để hiển thị các chỉ số thành phần. Thiết kế website đòi hỏi nhiều nguồn lực, cần kỹ năng lập trình nhiều hơn và khả năng nhắc người quản lý kiểm tra thông tin định kỳ. Vì vậy, dạng trình bày kết hợp theo gói hoàn chỉnh, chuyển thông tin qua thư điện tử hoặc các kênh thông tin xã hội (như Facebook, Twitter...) có thể phù hợp hơn. Với yêu cầu này, người có kỹ năng lập trình ở mức trung bình có thể thực hiện, mà không cần các chuyên gia về lập trình web.[5,6,7]

Vi vậy, nhóm nghiên cứu chọn ngôn ngữ $R$ để thiết kế bảng điều khiển kết hợp của bộ chỉ số chất lượng cốt lõi tại Bệnh viện Nhi Đồng 1, hướng đến sự tiện lợi và thân thiện với người dùng, cho cả người quản lý cấp bệnh viện, quản lý theo lĩnh vực (cấp phòng) và các khoa. 


\section{2. ĐỐI TƯớNG VÀ PHƯƠNG PHÁP}

Phương pháp nghiên cứu: Nghiên cứu triển khai (implementation research).

\section{Xây dựng bảng điều khiển:}

Về dữ liệu sử dụng để phân tích chỉ số: Dữ liệu giám sát quá trình (theo bảng kiểm giám sát chi tiết theo từng bước của quy trình chuyên môn) và đo lường kết quả được thu thập từ 3 nguồn chính: [a] Giám sát quá trình qua máy tính bảng trên ứng dụng dựa vào nền tảng miễn phí của ONA/ Enketo (định dạng tiêu chuẩn .xlsx hoặc .xlx); [b] Dữ liệu giám sát qua phiếu in (bảng kiểm hoặc bảng kiểm tra) và nhập liệu vào Excel; [c] Dữ liệu truy xuất từ hệ thống quản lý bệnh viện hoặc ki-ốt khảo sát cố định (định dạng Excel).

Chỉ số tuân thủ quá trình, sử dụng thuốc ngoại trú được phân tích bằng biểu đồ kiểm soát dành cho biến phân trăm có hiệu chỉnh Laney's (Laney's p-chart) và biểu đồ Laney's cho đại lượng u. Số lượt không hài lòng mỗi tuần qua máy khảo sát được phân tích bằng biểu đồ $\bar{X}-\bar{R}$.

Do có nhiều nguồn dữ liệu khác nhau, cấu trúc không thống nhất và quản lý phân mảnh, nhóm nghiên cứu biên tập dữ liệu dựa trên mã phân tích ngôn ngữ $R$ để chuẩn hóa thành dữ liệu mảng nhiều phân lớp. Sử dụng dữ liệu chuẩn hóa này để thiết lập bảng điều khiển. Dữ liệu đã được chuẩn hóa được tự động lưu trữ theo 2 định dạng .xlsx, .rda vào thư mục chỉ định để dự phòng (kiểm định và phục hồi dữ liệu). Toàn bộ dữ liệu truy xuất từ quan trắc gốc (bản sao) được sao lưu dự phòng ở thư mục được chỉ định trên hệ thống quản lý bệnh viện, phục vụ việc thẩm định chất lượng dữ liệu và xử lý sự cố về dữ liệu (nếu có).

Về ngôn ngữ lập trình ứng dụng và giao diện bảng điều khiển: Bảng điều khiển được thiết kế bằng ngôn ngữ $R$, trên giao diện tương tác người dùng của RStudio, dùng gói flexdashboard để thiết lập định dạng (layout) của bảng điều khiển, gói knitr để kết nối lệnh tự động trong $R$ Markdown.

Các gói biên tập và phân tích dữ liệu chính là: tidyverse (đơn giản cú pháp lệnh phân tích, dùng lệnh trong ggplot2 và sử dụng toán tử luồng “\%>\%"), readxl (đọc dữ liệu định dạng Excel tiêu chuẩn vì không có sẵn trong tidyverse), reshape2 (chồng các cột dữ liệu để lập dữ liệu mảng đa chiều), lubridate (xử lý các hàm thời gian), các gói thống kê biểu đồ quản lý chất lượng (qicharts2, qcc, ggQC), gridExtra (kết hợp biểu đồ), plotly (thêm thuộc tính interactive cho biểu đồ thiết kế trên nền tảng ggplot2), DT (lập bảng dữ liệu tổng hợp có chức năng tìm kiếm). [Mã phân tích của bảng điều khiển: liên lạc tác giả].

Đầu ra của $\mathrm{R}$ Markdown được chọn với định dạng .html nhằm dễ phổ biến. Nếu cần thiết có thể bổ sung thêm định dạng để thông tin trên mạng xã hội thường dùng như Facebook, Twitter). Tuy nhiên, do chỉ số chất lượng là thông tin mang tính nội bộ nên hiện tại nhóm nghiên cứu đang khóa định dạng đầu ra này.

\section{Đánh giá và hoàn thiện bảng điều khiển:}

Thiết kế nghiên cứu: Khảo sát cắt ngang qua bộ câu hỏi.

Đối tượng khảo sát: Gửi mẫu khảo sát cho toàn bộ 161 người dùng bảng điều khiển là các vị trí quản lý tại bệnh viện bao gồm ban giám đốc, trưởng khoa, trưởng phòng, điều dưỡng trưởng, phó khoa, phó phòng, thành viên mạng lưới quản lý chất lượng - an toàn người bệnh).

Nội dung khảo sát: Người dùng được hỏi ý kiến về mức độ tiếp cận và sự tiện lợi, hình thức trình bày phù hợp và mức độ dễ hiểu (xem chi tiết tại bảng 1). Thông qua tỷ lệ phản hồi để đánh giá một phần khả năng tiếp cận bảng điều khiển.

Phương pháp khảo sát: Lần 1 thực hiện qua phiếu tự điền (sau 2 tuần triển khai bảng điều khiển), lần 2 khảo sát trên biểu mẫu trực tuyến bằng cách gửi liên kết qua thư điện tử (sau 2 tuần cập nhật bảng điều khiển) đến toàn bộ người dùng do bệnh viện đang thực hiện giãn cách xã hội liên quan dịch Covid-19.

Phân tích dữ liệu: Tính tỷ lệ phản hồi và phần trăm các mức trả lời của người dùng theo từng 
nội dung. Ý kiến góp ý cụ thể được xem xét để điều chỉnh thiết kế, bổ sung nội dung, hướng dẫn kỹ năng cho người dùng nếu hợp lý.

Đánh giá mức độ tiết kiệm thời gian: Thống kê thực tế số giờ tiết kiệm được mỗi năm của các vị trí việc làm liên quan.

Vấn đề $\mathrm{y}$ đức nghiên cứu: Đề tài được Hội đồng thẩm định thông qua tại biên bản họp số 336/BB-BVND1 ngày 13-04-2020 và Giám đốc bệnh viện cho phép thực hiện tại Quyết định số 1128/QĐ-BVNĐ1 ngày 08-05-2020.

\section{KẾT QUẢ}

Cấu trúc và các chức năng thông tin của bảng điều khiển chỉ số: Bảng điều khiển chỉ số gồm có 10 phần được thiết kế theo thẻ (Tab): tóm tắt, kết luận, quy trình kỹ thuật, an toàn phẫu thuật, giám sát độc lập, dữ liệu, ki-ốt khảo sát không hài lòng (2 thẻ), thuốc ngoại trú và phòng ngừa ngã (xem hình 1). Biểu đồ Pareto chỉ rõ nội dung ít tuân thủ hoặc nhóm sự kiện bất lợi chiếm đa số được phân tích theo nhóm chỉ số kèm theo trong mỗi thẻ. Đối với quy trình kỹ thuật bác sĩ và điều dưỡng, do có rất nhiều quy trình chi tiết, việc phân tích được phân tầng theo khoa (hình 3a) và quy trình (hình $3 b$ ) dạng biểu đồ lưới để dễ thực hiện đối sánh giữa các khoa và quy trình, nhằm làm rõ vùng ưu tiên can thiệp. Biểu đồ kiểm soát đòi hỏi người dùng phải có kỹ năng nhất định để hiểu và phù hợp thứ bậc quản lý, nhóm bổ sung kết luận gồm 3 phần là [a] Kết luận (Nhận xét chung về chỉ số tuần hiện hành và vấn đề cốt lõi), [b] Bàn luận (Tóm tắt khuynh hướng và những vấn đề phát hiện trong tuần) và [c] Đềxuất (Nội dung cần xem xét cải tiến, tiến độ thực hiện và kết quả của những đề xuất ở tuần trước đó).

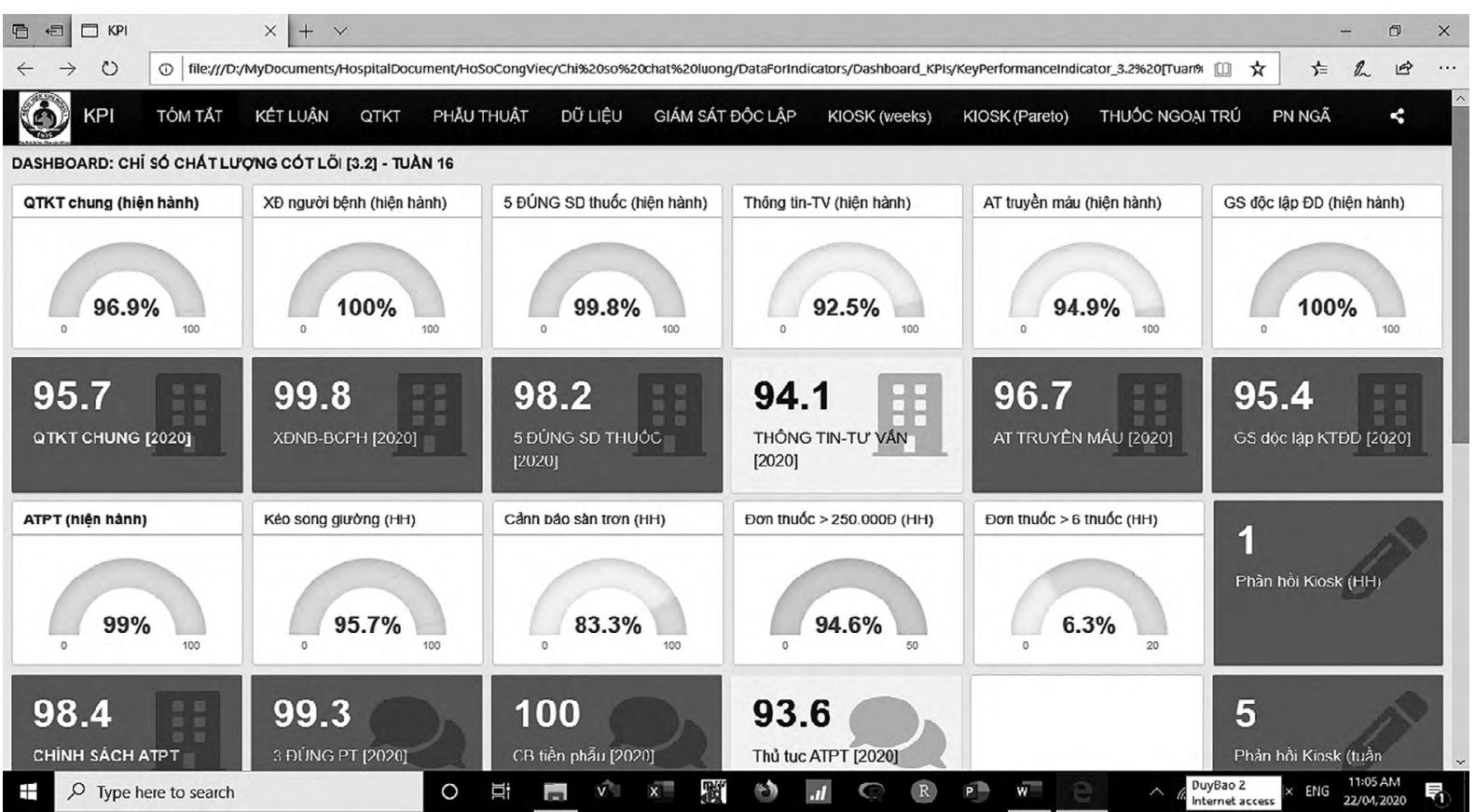

Hình 1. Trang đầu (tóm tắt) của bảng điều khiển chỉ số chất lượng

Biểu đồ kiểm soát chỉ số tuần được thiết kế theo nhóm gồm có: [a] Quy trình kỹ thuật (gồm: chỉ số chung, QTKT bác sĩ, QTKT điều dưỡng, các nội dung an toàn người bệnh theo điểm 1, điều 7 của Thông tư 19); [b] Chính sách an toàn phẫu thuật (tuân thủ chung, 3 đúng quanh phẫu thuật, chuẩn bị tiền phẫu, các thủ tục an toàn phẫu thuật); Dữ liệu (bảng tương tác cho phép lọc dữ liệu theo nhu cầu người dùng trước khi in hoặc tải về dưới định dạng Excel); [c] Giám sát độc lập (phân tích chỉ số 
chung toàn viện theo quy trình và theo khoa); và [f] Phòng ngừa ngã (cảnh báo sàn trơn và kéo [d] Kiosk khảo sát không hài lòng ở khu Khám song giường). Tính năng tương tác trên biểu đồ bệnh; [e] Sử dụng thuốc ngoại trú (tỷ lệ đơn thuốc giúp xem nhanh giá trị của các điểm dữ liệu trên trên 250.000 đồng và từ 6 loại thuốc trở lên) biểu đồ được người dùng quan tâm (xem hình 2).

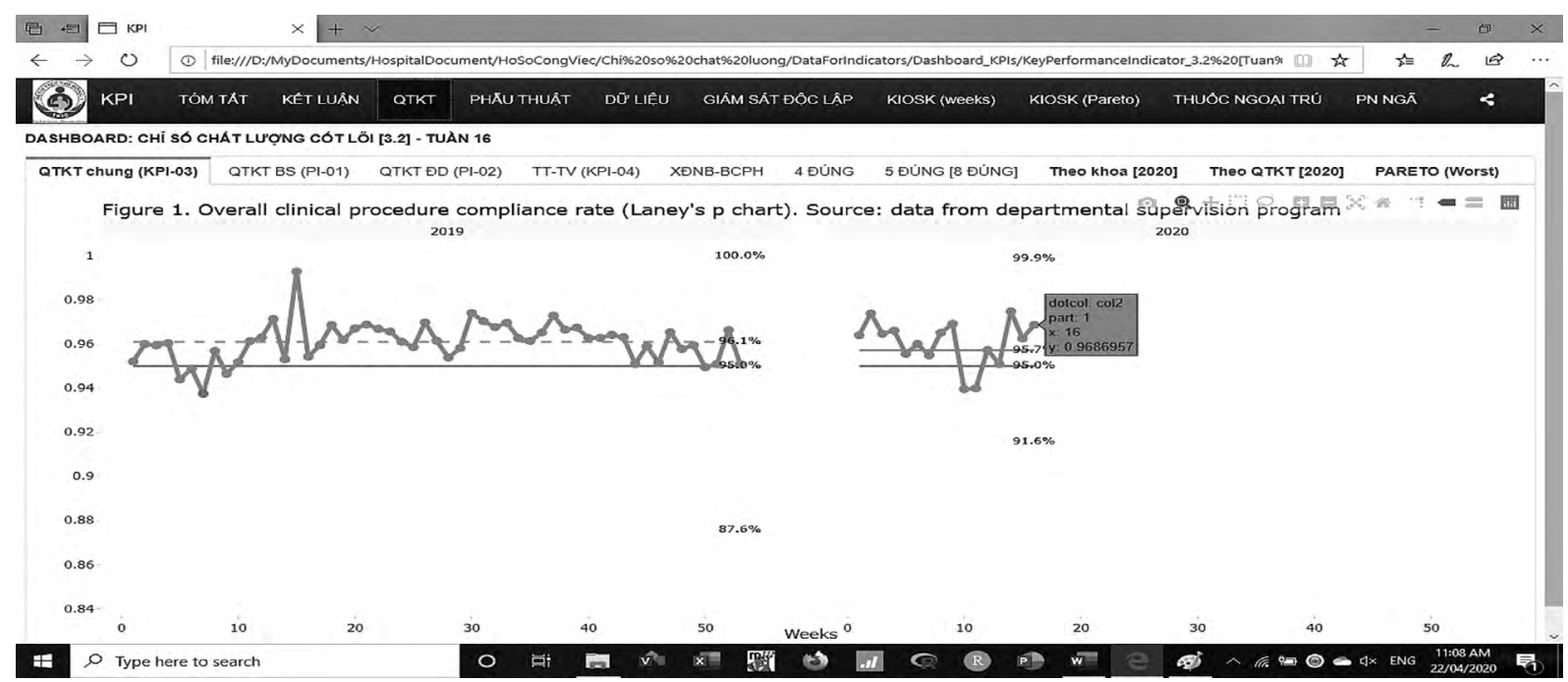

Hình 2. Minh họa nhóm biểu đồ kiểm soát có tính năng tương tác

Phân tích biểu đồ lưới với cảnh báo vi phạm quy luật biểu đồ kiểm soát theo khoa và quy trình cho phép truy vết nhanh chóng nguồn gốc của các biến động chỉ số chất lượng chung (hình $3 a$ và $3 b$ ). Đồng thời cũng giúp xác định nhanh chóng khoa hoặc quy trình nào không có dữ liệu giám sát, hoặc không thường xuyên.

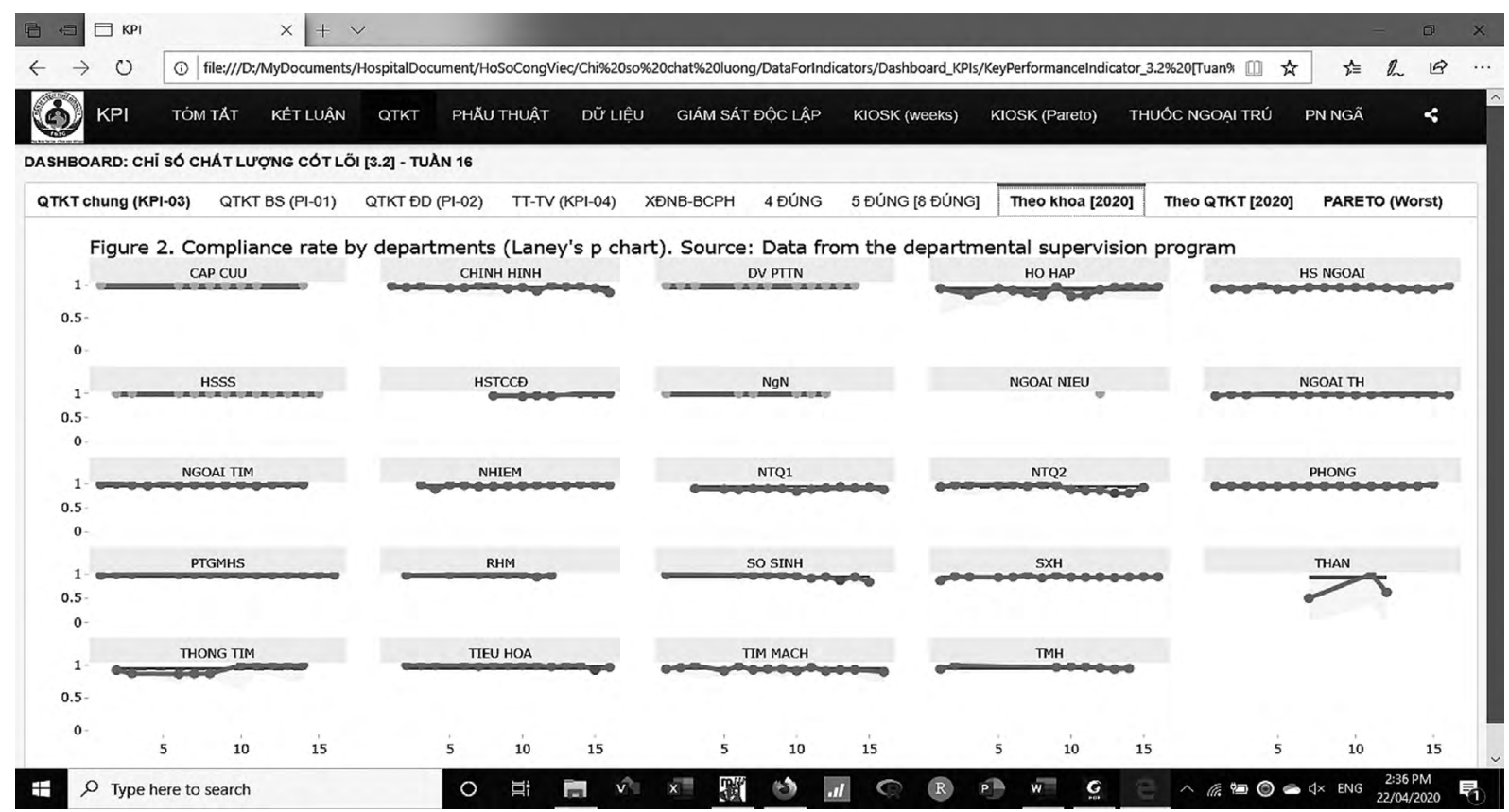

Hình 3a. Phân tích tỷ lệ tuân thủ quy trình kỹ thuật theo khoa 


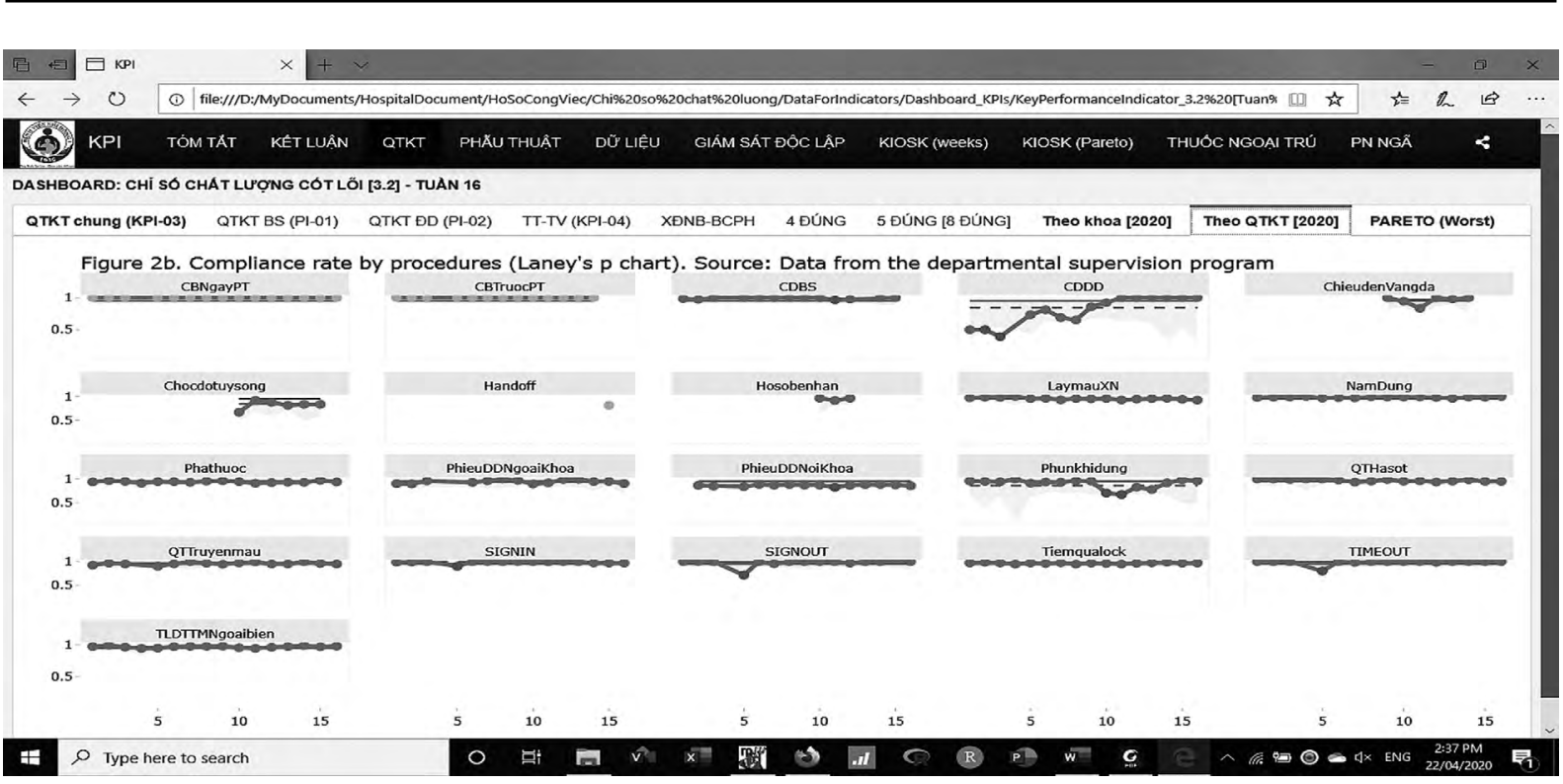

Hình 3b. Phân tích tỷ lệ tuân thủ quy trình kỹ thuật theo quy trình

Kết quả khảo sát ý kiến người dùng và điều chỉnh thiết kế bảng điều khiển:

Bảng điều khiển sơ bộ được phát triển và giới thiệu lần đầu ngày 26/12/2019. Sau 3 tuần thí điểm bản thiết kế ban đầu, nhóm nghiên cứu khảo sát ý kiến người dùng từ ngày 13-15/01/2020. Tỷ lệ phản hồi đợt khảo sát lần 1 là 19,9\%. Kết quả khảo sát được trình bày tại bảng 1 .

Ý kiến góp ý cụ thể ở lần khảo sát đầu tiên tập trung vào khả năng đăng nhập hộp thư bệnh viện, chưa hiểu nội dung biểu đồ, yêu cầu điều chỉnh để đọc bảng điều khiển chỉ số trên điện thoại thông minh (không đọc được) và yêu cầu tổ chức trang liên kết. Nguyên nhân do điện thoại chưa cài đặt ứng dụng Outlook, hộp thư bệnh viện cấp chưa được kích hoạt. Nhóm nghiên cứu đã hướng dẫn người dùng thực hiện các nội dung này. Riêng yêu cầu thiết lập trang liên kết nhóm không điều chỉnh vì định hướng phát triển bảng điều khiển kết hợp để dễ quản lý và phát hành thông tin, các trang liên kết (tính năng trong website) đã được thay thế bằng các thẻ (tab) có thể chuyển đổi để xem khá dễ dàng.

Bảng 1. Mức độ tiếp cận, sự thích hợp của dạng trình bày và mức độ dễ hiểu

\begin{tabular}{|c|c|c|c|c|}
\hline \multirow{2}{*}{ Nội dung khảo sát } & \multicolumn{2}{|c|}{ Lần 1 (n=32) } & \multicolumn{2}{|c|}{ Lần 2 (n=11) } \\
\hline & Lượt trả lời & $\%$ & Lượt trả lời & $\%$ \\
\hline \multicolumn{5}{|l|}{ Câu 1: Mức độ tiếp cận và nhận xét chung } \\
\hline Chưa xem lần nào & 5 & 15,6 & 0 & 0 \\
\hline Đã xem nhưng khó sử dụng & 9 & 28,1 & 0 & 0 \\
\hline Đã xem, thuận tiện khi sử dụng & 18 & 56,3 & 11 & 100 \\
\hline \multicolumn{5}{|l|}{ Câu 2: Hình thức trình bày phù hợp } \\
\hline Phù hợp & 21 & 75 & 10 & 90,9 \\
\hline Cần điều chỉnh & 7 & 25 & 1 & 9,1 \\
\hline Bất tiện & 0 & 0 & 0 & 0 \\
\hline \multicolumn{5}{|l|}{ Câu 3: Thông tin được cung cấp dễ hiểu } \\
\hline Dễ hiểu & 22 & 78,6 & 11 & 100 \\
\hline Khó hiểu & 6 & 21,4 & 0 & 0 \\
\hline Không hiểu được & 0 & 0 & 0 & 0 \\
\hline
\end{tabular}


Quá trình phát triển và hoàn thiện chỉ số qua nhiều phiên bản cùng với lượng giá không chính thức để hoàn thiện thiết kế. Phiên bản 2.0 (bổ sung phân tích chi tiết theo từng quy trình kỹ thuật, theo khoa dạng lưới và chức năng tìm dữ liệu theo khoa và quy trình) được giới thiệu ngày 16/3/2020. Phiên bản 3.1 (bổ sung đủ các chỉ số theo chủ đề an toàn người bệnh của Thông tư 19) được giới thiệu ngày $24 / 3 / 2020$, điều chỉnh lên phiên bản 3.2 (cấu trúc lại phần kết luận, bổ sung pereto nhóm quy trình kỹ thuật chung và phòng ngừa ngã theo đề nghị của Ban An toàn môi trường - An ninh trật tự') ngày 31/3/2020.

Lượng giá người dùng lần 2 (sau 3 tuần triển khai phiên bản 3.2) ngày 22-24/4/2020. Tỷ lệ phản hồi là $6,8 \%$. Kết quả lượng giá được trình bày tại bảng 1 cho thấy, tỷ lệ đồng thuận của người dùng có cải thiện rõ rệt dù cỡ mẫu khá nhỏ.

Thời gian tiết kiệm mỗi năm ước tính cho 23 người ở khoa lâm sàng (trung bình $1 \mathrm{giờ} / \mathrm{khoa} /$ tuần): $23 \times 52=1196$ giờ (tương đương 149,5 ngày công), đồng thời cũng đảm bảo chất lượng kết quả phân tích.

\section{BÀN LUÂN}

Bảng điều khiển kết hợp chỉ số chất lượng lần đầu được triển khai tại Bệnh viện Nhi Đồng 1. Một số hoạt động khác đã có triển khai dạng bảng điều khiển để quản lý hoạt động cụ thể như quản lý sử dụng máy thở, nhưng chưa có ứng dụng nào thiết lập bảng điều khiển kết hợp các biểu đồ chứa đựng nhiều phân tích thống kê khá phức tạp dùng trong quản trị chất lượng như biểu đồ kiểm soát (đủ các dạng) và biểu đồ Pareto.

Bảng điều khiển với hiệu ứng biểu đồ tương tác (interactive) cho phép người dùng có thể xem nhanh số liệu thống kê cụ thể từng điểm dữ liệu ngay trên biểu đồ, mà không cần phải hiển thị hết các số liệu, nhằm đảm bảo tính thẩm mỹ của các biểu đồ.

Bảng điều khiển được thiết kế chủ yếu cho người dùng máy tính cá nhân. Tuy nhiên, người dùng điện thoại thông minh (nếu cài đặt Outlook apps) hoàn toàn có thể sử dụng. Việc quyết định phạm vi phổ biến bảng điều khiển cho người dùng có địa chỉ thư điện tử nhidong.org.vn hoặc rộng rãi hoàn toàn có thể thực hiện theo chính sách quản lý chung của bệnh viện nhằm đảm bảo kiểm soát thông tin nội bộ.

Triển khai phân tích biểu đồ dạng lưới theo khoa và quy trình cho thấy lợi điểm để thực hiện truy vết nhanh nguồn gốc biến động của chỉ số chất lượng. Nó cũng có thể tạo ra một diễn đàn để đối sánh và làm tăng tính cạnh tranh giữa các khoa trong quá trình thực hiện cải tiến chất lượng.

Sáng kiến này cung cấp đủ bằng chứng để thực hiện đánh giá tiêu chí D3.2, đồng thời giúp tăng năng suất làm việc nhờ tiết kiệm được thời gian.

Điểm yếu của nghiên cứu này là cỡ mẫu khảo sát người dùng còn nhỏ, nên có thể thông tin thu được chưa phản ánh hết nhu cầu của họ. Nhưng với bản chất mở về mã nguồn, những yêu cầu phát sinh theo yêu cầu người dùng khá dễ bổ sung trong suốt quá trình sử dụng bởi người phụ trách bảng điều khiển.

\section{KẾT LUẬN}

Bảng điều khiển chỉ số không những giúp nhà quản trị bệnh viện dễ thấy bức tranh toàn cảnh về chất lượng, mà còn cung cấp đủ thông tin chi tiết để mỗi khoa, phòng thực hiện cải tiến.

Kiến nghị: Xu hướng triển khai thẻ điểm cân bằng trong quản lý chất lượng là cần thiết nếu thực sự muốn gắn liền hoạt động quản lý chất lượng với quản trị bệnh viện. Khi đó, bảng điều khiển chỉ số sẽ là một công cụ hữu dụng khi triển khai thẻ điểm cân bằng. Bảng điều khiển kết hợp được thiết kế trên ngôn ngữ $R$ có thể chuyển giao cho các cơ sở khác, với điều kiện đào tạo cơ bản ít nhất 1 người dùng có kỹ năng sử dụng $R$ ở trình độ cơ bản. Cần xem xét nhân rộng bảng điều khiển nhằm góp phần phát triển hoạt động quản lý chất lượng tại các bệnh viện. 


\section{TÀI LIỆU THAM KHẢO}

1. Bộ Y tế (2013). Thông tư số 19/2013/TT-BYT ngày 12/7/2013 của Bộ trưởng Bộ $Y$ tế hướng dẫn thực hiện quản lý chất lượng dịch vụ khám bệnh, chữa bệnh tại bệnh viện.

2. Bộ Y tế (2016). Tiêu chí chất lượng bệnh viện Việt Nam, phiên bản 2.0 ban hành theo Quyết định số 6858/QĐ-BYT ngày 18/11/2016 của Bộ trưởng Bộ $Y$ tế.

3. Tổng cục Đo lường chất lượng (2016). Tiêu chuẩn Việt Nam TCVN ISO 9001:2015 (phiên bản 4).

4. DataCampTeam (2020). Choosing Python or R for Data Analysis? An Infographic: https://www. datacamp.com/community/tutorials/r-or-python -for-data-analysis.

5. Anne Marie JWM, Weggelaar - Jansen, Damien SE, Broekharst, Martine de Bruijne
(2018). Developing a hospital - wide quality and safety dashboard: a qualitative research study. BMJ Qual Saf; 27: 1000-1007. doi: 10.1136/ bmjqs - 2018-007784 .

6. Diego AM, Erin MK, Mehdi J, James C, Hetal R, Rohit T, Charles B, Bree B, Scott R, Levin (2018). An Electronic Dashboard to Monitor Patient Flow at the Johns Hopkins Hospital: Communication of Key Performance Indicators Using the Donabedian Model. Journal of Medical Systems, 42: 133; https:// doi.org/10.1007/s10916-018-0988-4.

7. Rebecca R, Natasha A, Lynn M, Roy AR, Dawn D (2016). Requirements for a quality dashboard: Lessons from National Clinical Audits. NIHR project number 16/04/06. 